\title{
Object-Oriented Database Model For Effective Mining Of Advanced Engineering Materials Data Sets
}

\author{
Doreswamy ${ }^{1}$, Manohar $\mathrm{M} \mathrm{G}^{2}$, Hemanth $\mathrm{K} \mathrm{S}^{3}$ \\ ${ }^{1,2,3}$ Department of Computer Science \\ Mangalore University, Mangalagangotri-574 199, Karnataka, INDIA, \\ doreswamyheyahoo.com, \\ manohargo@gmail.com, reachhemanthmca@gmail.com
}

\begin{abstract}
Materials have become a very important aspect of our daily life and the search for better and new kind of engineered materials has created some opportunities for the Information science and technology fraternity to investigate in to the world of materials. Hence this combination of materials science and Information science together is nowadays known as Materials Informatics. An Object-Oriented Database Model has been proposed for organizing advanced engineering materials datasets.
\end{abstract}

\section{Keywords}

Engineering Materials, Materials Informatics, Object Oriented Database Model.

\section{INTRODUCTION}

Engineering materials databases are the basis of materials informatics, manufacturing industries and the related disciplines[1]. Design and development of materials databases is done to enrich the availability and accessibility of materials data to materials scientist, researcher and design engineers in manufacturing industries[2]. Many manufacturing industries have organized the materials data into databases and that are being accessed to search and screen materials that are suitable for design requirements. The International Atomic Energy Agency (IAEA) has created a database of natural matrices that is internationally available as certified reference materials (CRM). This database is accessing frequently and updated periodically, but its accessibility is limited to developmental work of IAEA.

Data mining is the discovery of knowledge and useful information from the large amounts of data stored in databases[3]. With the increasing popularity of object-oriented database systems in advanced database applications, it is important to study the data mining methods for objectoriented databases because mining knowledge from such databases may improve understanding, organization, and utilization of the data stored there.

\section{THE OBJECT-ORIENTED DATABASE (OOD) MODEL}

Object-oriented database systems, which can be considered fifth-generation database technology, began developing in the mid-80's out of a necessity to meet the requirements of applications beyond the data processing applications, which characterized relational database systems (fourthgeneration database technology). Attempts to use relational database technology for advanced

David C. Wyld, et al. (Eds): CCSEA, SEA, CLOUD, DKMP, CS \& IT 05, pp. 129-137, 2012.

(C) CS \& IT-CSCP 2012

DOI : $10.5121 /$ csit.2012.2214 
applications like Computer Aided Design (CAD), Computer Aided Manufacturing (CAM), software engineering, Knowledge-Based Systems, and Multimedia Systems, quickly exposed the shortcomings of relational database systems. The need to perform complex manipulations on existing databases and a new generation of database applications generated a need that would be better satisfied by object-oriented databases (OODBs).

In the object-oriented data model (OODM), any real world entity is represented by only one modeling concept - the object. An object has a state and behaviour associated with it. The state of an object is defined by the value of its properties (attributes). Properties can have primitive values (like strings and integers) and non-primitive objects. A non-primitive object would in turn consist of a set of properties. Therefore objects can be recursively defined in terms of other objects. The behaviour of an object is specified by methods that operate on the state of the object. Each object is uniquely identified by a system-defined identifier. Objects with the same properties and behaviour are grouped into classes. An object can be an instance of only one class [4] or an instance of several classes.

Classes are organized in class hierarchies. A subclass inherits properties and methods from a super class, and in addition, a subclass may have specific properties and methods. In some systems, a class may have more than one super class (multiple inheritance), while in others it is restricted to only one super class (single inheritance).

Most models allow for overriding inherited properties and methods. Overriding is the substitution of the property domain with a new domain or the substitution of a method implementation with a different one [5].

\section{GENERAL OOD MODEL FOR ADVANCED ENGINEERING MATERIALS DATA}

Advanced engineering materials are high performance, reliable, durable and performance driven, application oriented materials. These materials are designed and synthesised with the combinations of basic engineering materials with reinforcement materials. There are more than 50,000 advanced engineering materials and their applications are richly enhanced in various science and technology fields. Database technology is proposed for organizing these materials and their properties and relationships with other materials[6]. The use of database technology in the engineering design applications differs from traditional data processing. Many database features developed for the more traditional applications can be transferred to the engineering design environment. Some of these features take on new meaning, but many are only viewed from a different perspective in engineering design applications. Engineering design applications impose unique requirements on, but also can particularly benefit from, the data representation, Data definition and data manipulation processing aspects of database technology.

Many approaches have been taken in the computer-based organization and delivery of engineering materials data and expertise[7], but relatively little attention has been given to the provision of information to designers working in adaptive or variant design areas in which only incremental design changes are made. The information required is characterized by the variety of its formats and Model, and by the complexity of the interrelationships between data. Object Oriented Model $(\mathrm{OOM})$ is proposed for organizing the advanced entering materials data and their properties relation with other materials. The purpose of the data model is to bring the design of a database that performs efficiently, retains correct information whose logical structure is natural enough to be understood by and is as easy as possible to maintain and extend. 


\subsection{Object Oriented Features With Relevance To Advanced Engineering Materials}

\begin{tabular}{|l|}
\hline \multicolumn{1}{|c|}{ Matrix } \\
\hline +Mat_Name \\
+Mat_Type \\
+Mat_Prop \\
\hline +CreateMaterial(MatName, PropertiesValues, MatType) \\
+DestroyMaterial(Obj) \\
+SetMatName(MatName) \\
+GetMatName(Obj) \\
+SetMatType(MatType) \\
+IsComposite(Obj) \\
+IsMetal(Obj) \\
+IsPolymer(Obj) \\
+IsCeramic(Obj) \\
\hline
\end{tabular}

Fig. 1. Sample Class Diagram

Class: An object class describes a group of objects with similar properties (attributes), common behaviour (Operations), common relationship to other objects and common semantics. Often the word 'class' is used instead of 'object class'. an attribute is a data value held by the object in a class. An operation is a function that may be applied to or by objects in a class. The figure 1 shows a class matrix with attributes and its operations.

Instantiation: It is the relationship between an object and its class. The notation for instantiation is a dashed line from the instance to the class with an arrow pointing to the class.; the dashed line is ladled with legend instance enclosed by $(<<>>)$.

Links and Association: A link is a physical or conceptual connection between object instances for eg., material-1 has property-1. Mathematically a link is defined as a tuple, that is, an ordered list of object instances. An association describes a group of links with common structure and common semantics.

Aggregation: It is the "part-whole" or "a-part-of" relationship in which objects representing the components of something are associated with an object representing the entire assembly. The aggregation is a special form of association.

Abstract and Concrete Class: An abstract class is a class that has no direct instances but whose descendent classes have direct instances. A concrete class is a class that is instantiable, i.e., it can have direct instances.

Generalisation, Specialisation and Inheritance: Generalisation is the relationship between a class and one or more refined versions of it. The class being refined is called the superclass and each refined version is called a subclass.

Superclass: The basic attributes and operations common to a group of subclasses are attached to the superclass and shared by each subclass. Each subclass is said to inherit the features of its superclass. Each subclass not only inherits all the features of its ancestors but adds its own specific attributes and operations as well.

\subsubsection{Advantages of OODB}

a) Objects in an OODBMS can store an arbitrary number of atomic types as well as other objects. The fact that a OODBMS is better suited to handling complex, interrelated data than a Relational Database Management systems(RDBMS) means that an OODBMS can outperform a RDBMS by ten to a thousand times depending on the complexity of the data being handled. 
b) A query language is not necessary for accessing data from an OODBMS unlike a RDBMS since interaction with the database is done by transparently accessing objects. It is still possible to use queries in an OODBMS however.

c) In a typical application that uses an object oriented programming language and a RDBMS, a significant amount of time is usually spent mapping tables to objects and back. This "impedance mismatch" is completely avoided when using an OODBMS.

d) The user of a RDBMS has to worry about uniquely identifying tuples by their values and making sure that no two tuples have the same primary key values to avoid error conditions.

e) With a RDBMS it is not possible to model the dynamic operations or rules that change the state of the data in the system because this is beyond the scope of the database. With an OODBMS there is no disconnect between the database model and the application model because the entities are just other objects in the system.

\subsubsection{Disadvantages of OODB}

a) Schema Changes: In a RDBMS modifying the database schema either by creating, updating or deleting tables is typically independent of the actual application. In an OODBMS based application modifying the schema by creating, updating or modifying a persistent class typically means that changes have to be made to the other classes in the application that interact with instances of that class. This typically means that all schema changes in an OODBMS will involve a system wide recompile. Also updating all the instance objects within the database can take an extended period of time depending on the size of the database.

b) Language Dependence: An OODBMS is typically tied to a specific language via a specific API. This means that data in an OODBMS is typically only accessible from a specific language using a specific API, which is typically not the case with a RDBMS.

c) Lack of Ad-Hoc Queries: In a RDBMS, the relational nature of the data allows one to construct ad-hoc queries where new tables are created from joining existing tables then querying them. Since it is currently not possible to duplicate the semantics of joining two tables by "joining" two classes then there is a loss of flexibility with an OODBMS. Thus the queries that can be performed on the data in an OODBMS is highly dependent on the design of the system.

\subsection{Object -Oriented Database For Engineering Materials Data Set}

After considering all the above aspects of Object Oriented Modeling, a database architecture is designed as shown in the figure 2 . Wherein the all properties of the materials are segregated in to 11 different property classes based on the characteristic of the property. eg., Mechanical Properties are grouped in to MechProp class, Chemical properties in to ChemProp class and Thermal properties in to ThrmProp class and so on.

All these 11 classes are aggregated to the 'Property class' which is the super class for the Matrix and ReinforcementMaterial classes. This property class is completely dependent on the 11 property-classes, as its attributes are the objects of those 11 classes.

Each of these classes have their own behaviours (Operations) to operate on the attributes of their respective classes. For eg., SetThrmProp() and GetThrmProp are the operations of the ThrmProp Class to set data to or read data from the object respectively. Moreover these classes can have some specific functions for computation and prediction of attribute values of the objects. The Matrix combined together with Reinforcement material creates a ReinforcedCompositeMaterial hence becomes an example of multiple inheritance. 


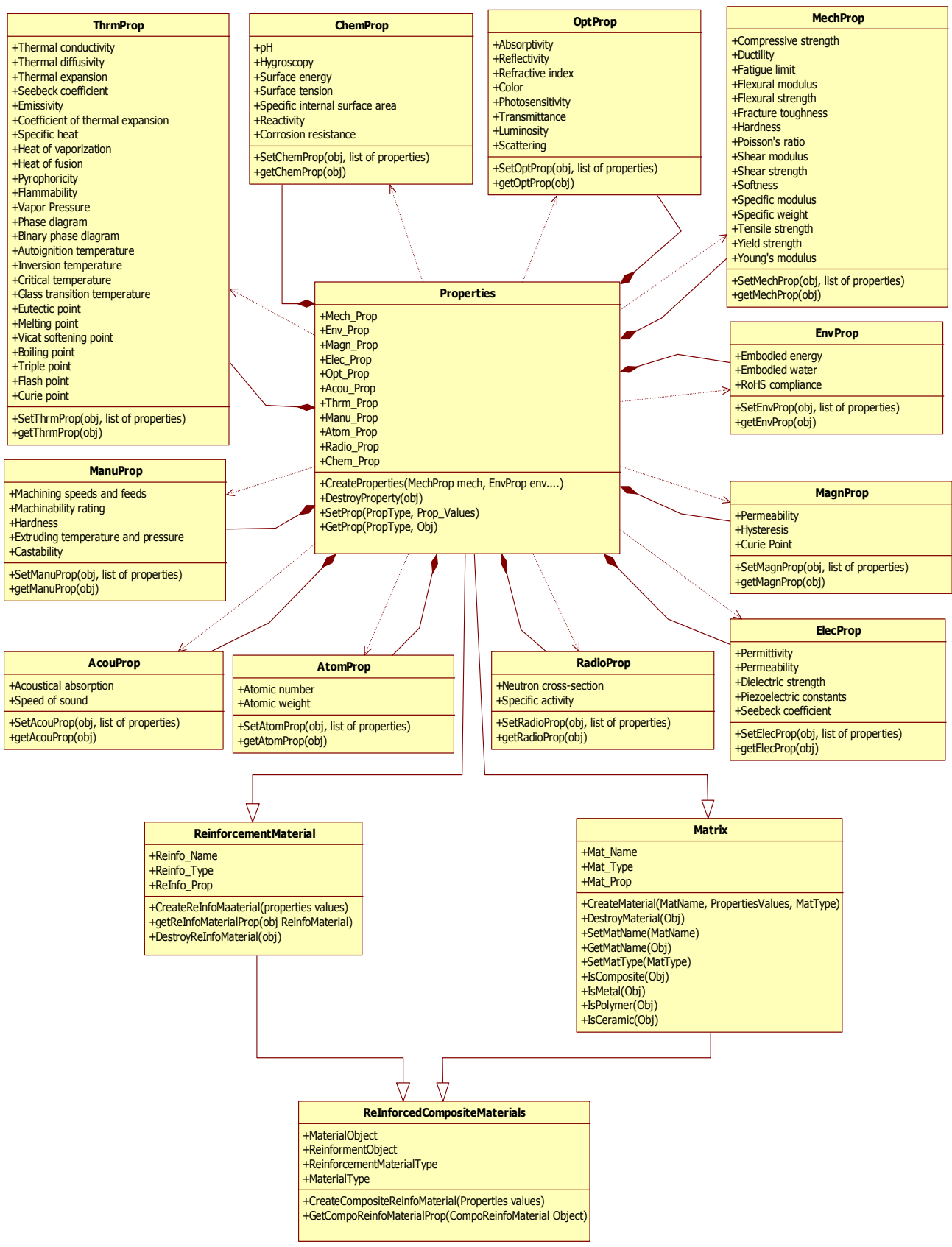

Figure 2: The object oriented database Structure of the Material Database

\subsection{Reinforcement Materials}

The reinforcement materials are divided into 3 main parts as small, medium and long materials depending on their physical appearance. The inter composition of these 3 reinforcement materials gives 7 types of new reinforcement materials. The following figure 3 illustrates different Reinforcement materials classes inherited from reinforcement class. 


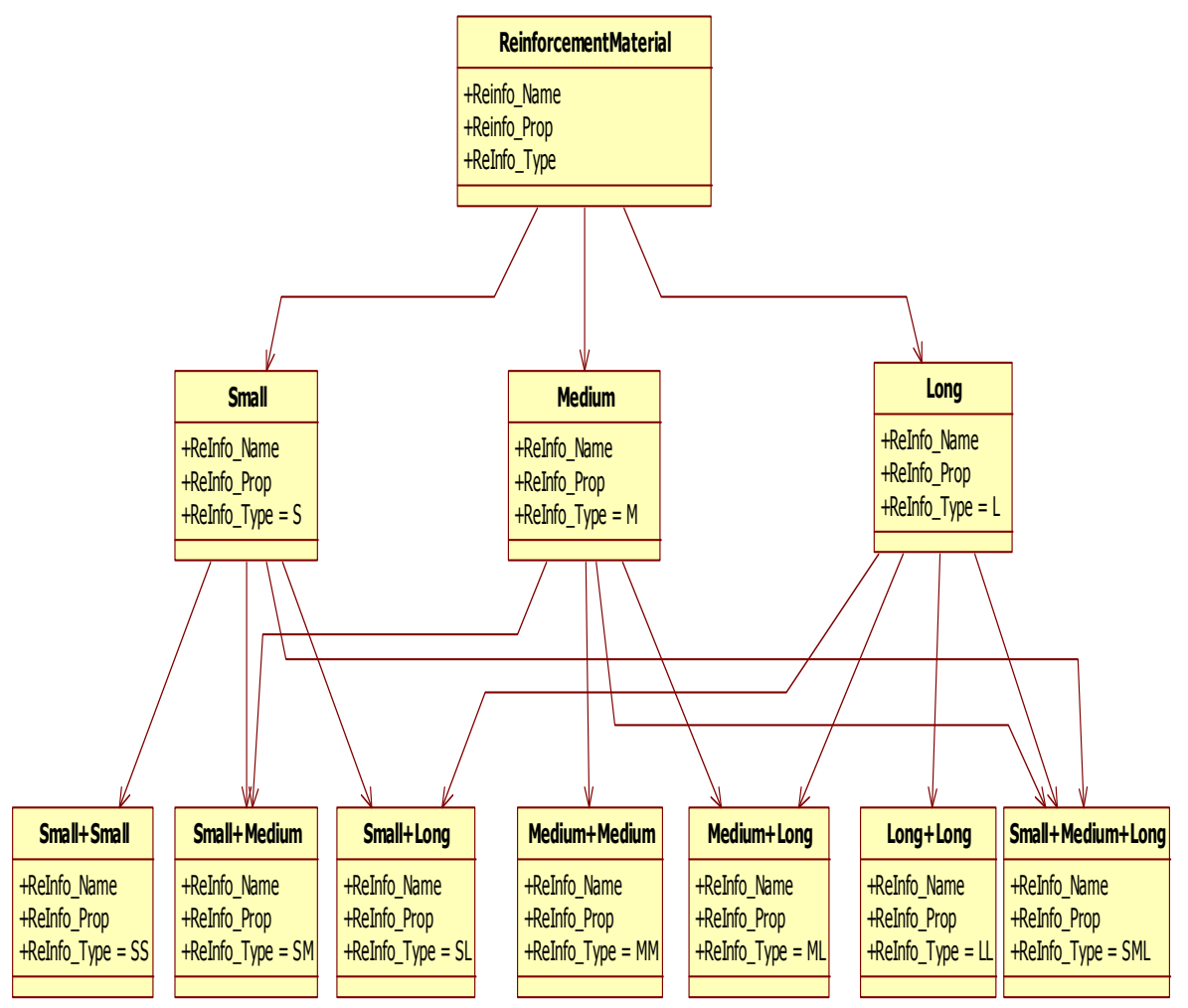

Figure 3: Different Reinforcement materials Classes inherited from reinforcement class

\subsection{Composite Materials}

Composites are made up of individual materials referred to as constituent materials by grouping two more constituent materials together. There are basically 3 types of constituent materials namely Metals, Ceramics and polymers. These materials are grouped together to produce the composite of different kinds as shown in the figure 4. 


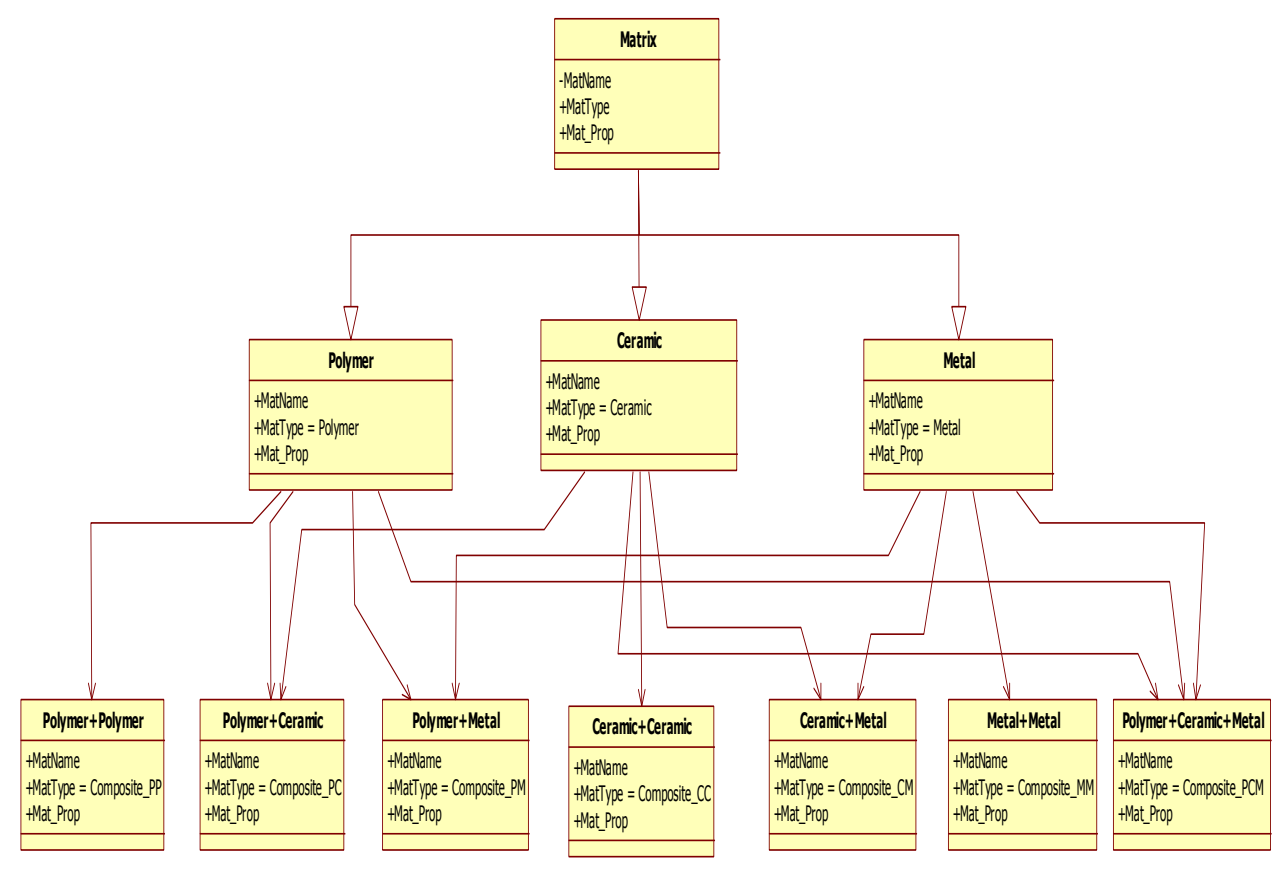

Figure 4: Different Material Classes inherited from abstract class Matrix

\subsection{Reinforced Composite Materials}

Reinforced Composites are made up of individual constituent materials, composite materials and reinforcement. There are two categories of constituent materials: matrix and reinforcement. At least one portion of each type is required. The matrix material surrounds and supports the reinforcement materials by maintaining their relative positions. The reinforcements impart their special mechanical and physical properties to enhance the matrix properties. This produces a material with properties which are unavailable from the individual constituent materials, while the wide variety of matrix and strengthening materials allows the designer of the product or structure to choose an optimum combination. This can be shown the figures 5 


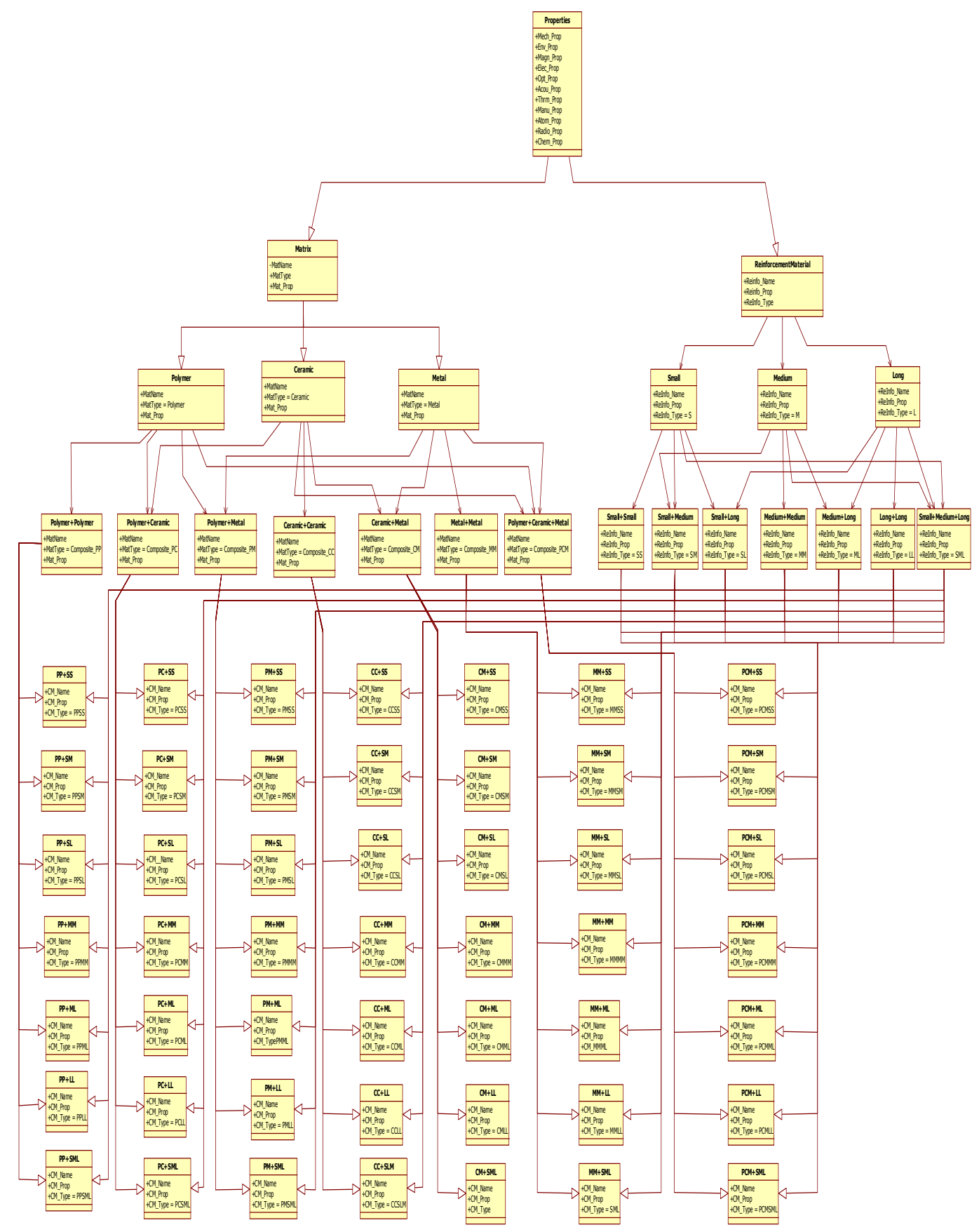

Figure 5: Different possible Composite materials Classes inherited from both matrix and reinforcement classes.

\section{CONCLUSION AND FUTURE SCOPE}

The object oriented model is employed to engineering materials data organization. Issues on generalization-based data mining in object-oriented databases are investigated in three aspects: (1) generalization of complex objects such as Matrix, Properties and Reinforcement and 
Composite materials Classes, (2) class-based generalization that include polymer, ceramic and metal classes, and (3) enumeration of different kinds of objects of composite types.

Further, sophisticated rule formation methods can be developed for extraction of different kinds of knowledge from data, including characteristic rules, discriminate rules, association rules, and classification rules. The application of such discovered knowledge may substantially enhance the power and flexibility of browsing databases, organizing databases and querying data and knowledge in the advanced engineering object-oriented databases.

\section{ACKNOWLEDGMENTS}

This work has been supported by the University Grant Commission(UGC), India under Major Research Project entitled "Scientific Knowledge Discovery Systems (SKDS) For Advanced Engineering Materials Design Applications" vide reference F.No. 34-9912008 (SR), 30th December 2008. The authors gratefully acknowledge the support and thank the authors.

\section{REFERENCES}

[1] Hrubiak.R., George,L., Saxena,S.K., Rajan, K., "A Materials Database for Exploring Material Properties", Journal of Materials, Vol. 61, pp. 59-62, (2009).

[2] Doreswamy, "A Survey For Data Mining Framework For Polymer Matrix Composite Engineering Materials Design Applications", International Journal of Computational Intelligence Systems (IJCIS), Vol. 1. Issues 4, pp.312-328, (2008).

[3] Jiawei Han, Shojiro Nishio, Hiroyuki Kawano, Wei Wang, "Generalization-based data mining in object-oriented databases using an object cube model", Data \& Knowledge Engineering - Special jubilee issue: DKE 25 archive Volume 25 Issue 1-2, March 1998

[4] Michael Dobrovnik and Johann Eder, "View Concepts for Object-Oriented Databases", Advances in Cognitive Engineering and Knowledge-Based Systems, Proc. of the Intl. Symposium on Systems Research, Informatics and Cybernetics, Baden-Baden,1993

[5] Babak Ameri Shahrabi, Wolfgang Kainz, "An Implementation Approach for Object-oriented Topographic Databases using Standard Tools", United Nations University on March 5, 2008

[6] Xia Li, Hang Su, Xiaoling Chen, Caifu Yang, Gang Xie, The Development Of A Materials Database In China, Data Science Journal, Volume 6, Supplement, (2007).

[7] Wei Qunyi, PENG Xiaodong, LIU Xiangguo, XIE Weidong.: "Materials informatics and study on its further development". Chinese Science Bulletin. vol.51,pp. 498-504(2006). 